\title{
Examining the Sensitivity of Electron Holography for Two-Dimensional p-n Junction Mapping
}

Zhouguang Wang and Du Li

Micron Technology, Inc., Boise, ID 83707

The shrinkage of the process geometry in the semiconductor industry requires a technique to obtain two-/three-dimensional (2D/3D) dopant profiles, with high spatial resolution and wide dynamic sensitivity, to understand device performance. Off-axis electron holography (EH) based on transmission electron microscopy (TEM) has been demonstrated to be capable of mapping and characterizing 2D electrostatic potential distributions, and hence, the doping profiles in devices with nanometer resolution [1]. However, very little is known about the sensitivity of electron holography in dopant profiling. To develop the $\mathrm{EH}$ technique for practical use, it is necessary to systematically examine the sensitivity.

Recently, it has been reported that a Si/Si p-n junction implanted in a lower level $\left(10^{15}\right.$ $\mathrm{cm}^{-3}$ ) p-wafer was successfully mapped and characterized by EH [2]. In current work, a Si/Si p-n junction serial with different $\mathrm{n}+$-doping levels on highly doped $\mathrm{p}$-wafers was fabricated for $\mathrm{EH}$ examination. The p-wafer is $\sim 10^{19} \mathrm{~cm}^{-3}$ boron (B)-doped Si (100) (sub-Si). After epitaxially growing a $50 \mathrm{~nm}$ intrinsic $\mathrm{Si}$ layer (EPI) on the sub-Si, a $50 \mathrm{~nm}$ silicon oxide $\left(\mathrm{SiO}_{2}\right)$ layer was deposited. Phosphorus (P) ions were then implanted into $\mathrm{SiO}_{2}$ layer at $40 \mathrm{keV}$ with four different doses: $1 \times 10^{14}, 1 \times 10^{13}, 1 \times 10^{12}$ and $1 \times 10^{11} \mathrm{~cm}^{-2}$. The $n$-type surface layers with different doping levels were formed on the sub-Si by rapid thermal annealing (RTP) at $850^{\circ} \mathrm{C}$ for 10 seconds. Secondary-ion mass spectrometry (SIMS) was used to obtain depth profiles and concentrations of the dopants in the samples. Fig. 1(a) gives depth distributions of $\mathrm{P}$ and $\mathrm{B}$ for the sample with the implanting dose of $1 \times 10^{13} \mathrm{~cm}^{-2}$. The $\mathrm{P}$ depth profiles in all four samples are shown in Fig. 1(b), which indicates that concentrations of $\mathrm{P}$ around the EPI/sub-Si interface are $\sim 4 \times 10^{18}$, $4 \times 10^{17}$ and $4 \times 10^{16} \mathrm{~cm}^{-3}$, respectively, for the samples with implanting doses of $1 \times 10^{14}, 1 \times 10^{13}$ and $1 \times 10^{12} \mathrm{~cm}^{-2}$. For the sample with implanting dose of $1 \times 10^{11} \mathrm{~cm}^{-2}$, the $\mathrm{P}$ concentration around the EPI/sub-Si interface is lower than the detection limit $\left(\sim 1 \times 10^{16} \mathrm{~cm}^{-3}\right)$ of the SIMS.

Cross-sectional TEM samples were prepared by focus ion beam (FIB) operating at 30 $\mathrm{keV}$. EH experiments were performed on a JEOL 2010F TEM equipped with an electron biprism. To get a proper field of view, a Gatan Image Filter (GIF) was used to obtain extra magnification and better signal-to-noise ratio. Digital holograms were reconstructed using the Holoworks plugin for Gatan DigitalMicrograph software. In Fig. 2, holograms (a), (c), and (e) are taken from three samples with lower implanting doses: $1 \times 10^{11}, 1 \times 10^{12}$ and $1 \times 10^{13} \mathrm{~cm}^{-2}$, respectively. The reconstructed phase images corresponding to the holograms are shown in Figs. 2(b), 2(d) and 2(f). In the phase images, there is an apparent phase jump in the $\mathrm{SiO}_{2} / \mathrm{Si}$ interface. In the $\mathrm{Si}$ region, the n-type surface layer is distinguished clearly from the p-region by brighter contrast, even for the sample with the lowest doping level $\left(1 \times 10^{16} \mathrm{~cm}^{-3}\right.$ or less). This work demonstrates that electron holography has not only high spatial resolution but also high sensitivity for 2D dopant profiling.

References

[1] W.D. Rau et al., Phys. Rev. Letts. 82 (1999) 2614.

[2] Z. Wang et al., Appl. Phys. Lett. 81 (2002) 478. 

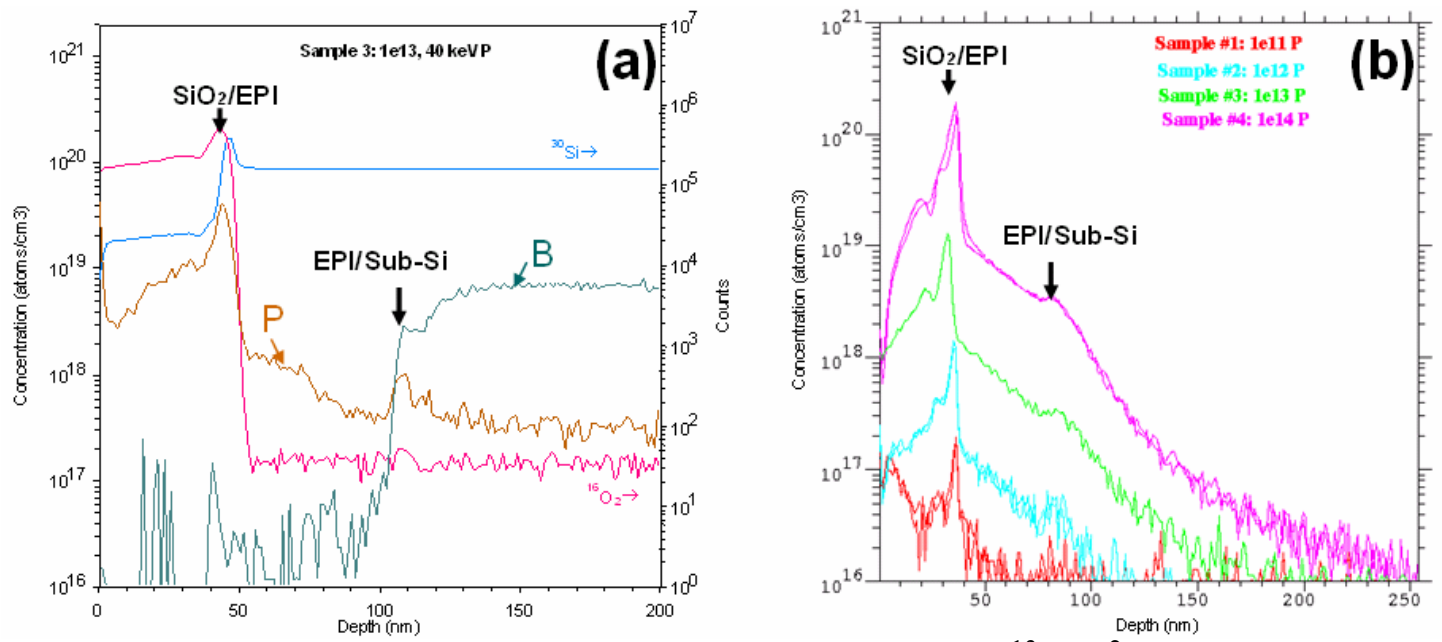

Fig. 1 (a) SIMS results from the sample implanted at dose $1 \times 10^{13} \mathrm{~cm}^{-2}$; (b) $\mathrm{P}$ doping profiles for all four samples implanted at different doses.
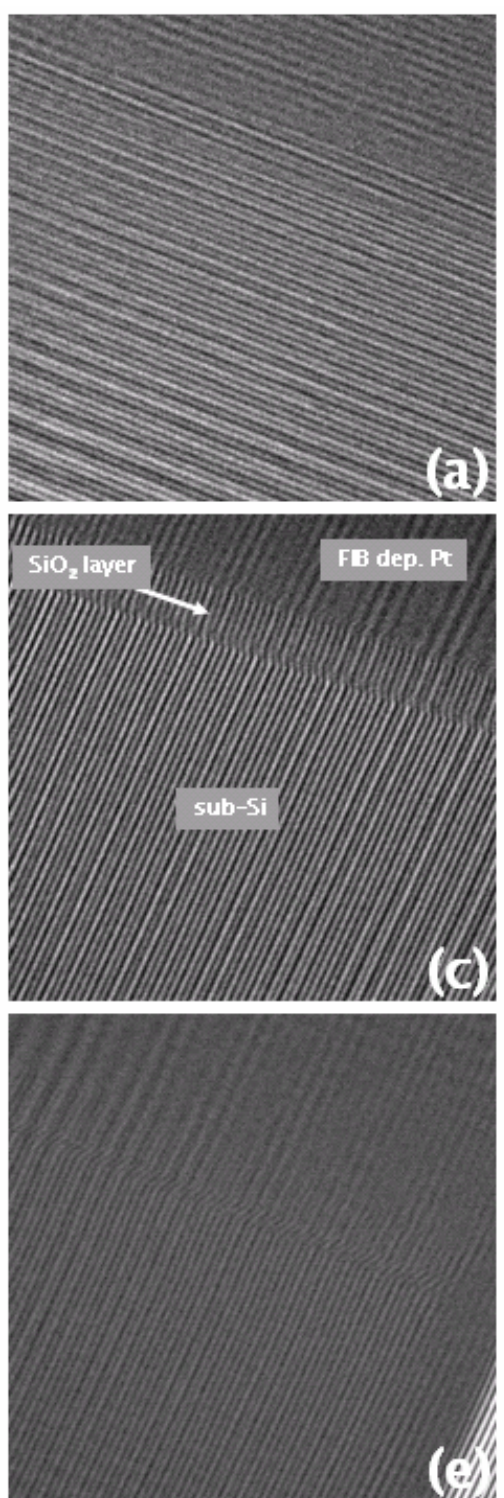
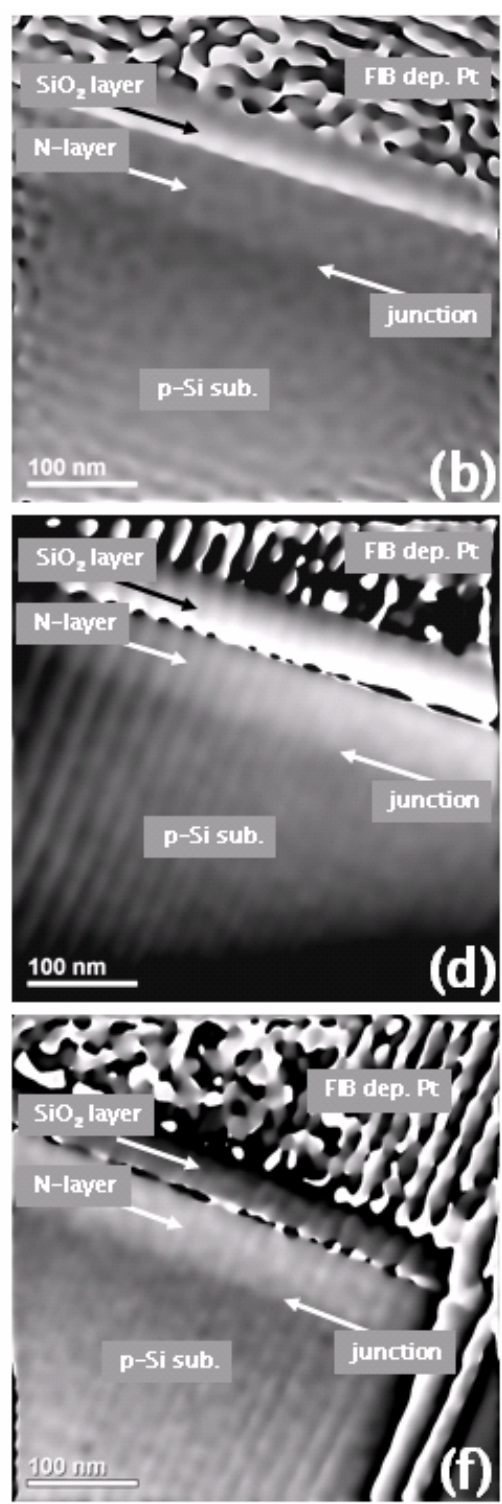

Fig. 2 Electron holograms (a), (c), and (e) and corresponding reconstructed phase images (b), (d), and (f), respectively, for samples implanted at different doses:

(a) and (b) -- $1 \times 10^{11} \mathrm{~cm}^{-2}$;

(c) and (d) $--1 \times 10^{12} \mathrm{~cm}^{-2}$;

(e) and (f) $--1 \times 10^{13} \mathrm{~cm}^{-2}$. In the phase images, $p-n$ junction contrast is clearly visible. 